\title{
Quantum escape kinetics over a fluctuating barrier
}

\author{
Pulak Kumar Ghosh ${ }^{2}$, Debashis Barik ${ }^{2}$, Bidhan Chandra Bag ${ }^{1}$ and Deb Shankar Ray ${ }^{*}$ \\ Indian Association for the Cultivation of Science, Jadavpur, Kolkata 700 032, India
}

\begin{abstract}
The escape rate of a particle over a fluctuating barrier in a double well potential exhibits resonance at an optimum value of correlation time of fluctuation. This has been shown to be important in several variants of kinetic model of chemical reactions. We extend the analysis of this phenomenon of resonant activation to quantum domain to show how quantization significantly enhances resonant activation at low temperature due to tunneling.
\end{abstract}

\section{INTRODUCTION}

The enhancement of small periodic signal by noise in a nonlinear system has been a theme[1] of topical interest over more than two decades. Ever since the observation of this phenomenon of stochastic resonance in varied theoretical and experimental contexts, a number of noise-induced resonance effects have been reported [2, 3, 4, 5, 6, 7]. A prototypical effect of this kind, known as resonant activation discovered by Doering and Godua [14], concerns a resonance effect in the escape rate of a particle over a fluctuating barrier in a bistable potential, where the resonance 15, 16, 17, 18, 19, 20, 21, 22, 25 can be achieved by observing the variation of mean first passage time as function of flipping rate of fluctuation of the barrier height. In the simplest possible term the phenomenon can be realized in a model with a linear barrier with a slope which fluctuates between two values. The phenomenon has triggered a lot of theoretical activity around the Markovian and non-Markovian variants of kinetic models for chemical reactions [15, 16, 17, 19] and several related issues and been experimentally observed by Mantegna and Spagnolo [22]. This fluctuation of potential is also important in various problems of chemical physics, notably in molecular dissociation dynamics 23], protein folding 24] among others. The purpose of this paper is to extend the analysis of resonant activation to quantum domain. Since at low temperature thermal activation is accompanied by tunneling, the question that naturally arises is how noise-induced resonance effects manifest themselves in a quantum system. Specifically our object here is two-fold: First, we intend to understand the counterintuitive role of external noise in bringing out the resonance behaviour in a bistable quantum system where the mean escape time is varied as a function of correlation time of noise. Second, it is worthwhile to examine the nature of this resonance in presence of generic quantum effects like tunneling at low temperature and allow ourselves a fair comparison with the classical results. In what follows we carry out a theoretical and a numerical study of quantum stochastic dynamics of a bistable system with a barrier height fluctuating due to an external Ornstein-Uhlenbeck noise and show how the

\footnotetext{
*e-mail address: pcdsr@mahendra.iacs.res.in
}

mean escape time is profoundly influenced by the statistical properties of the noise particularly the correlation time in exhibiting the quantum resonance activation.

\section{QUANTUM STOCHASTIC DYNAMICS}

\section{A. General aspects}

To derive quantum Langevin equation from a microscopic picture consider the well-known standard system-reservoir model with following form of the Hamiltonian 35$]$

$$
\hat{H}=\frac{\hat{p}^{2}}{2 m}+V(\hat{x}, t)+\sum_{j=1}^{N}\left\{\frac{\hat{p}_{j}^{2}}{2}+\frac{1}{2} \kappa_{j}\left(\hat{q}_{j}-\hat{x}\right)^{2}\right\}
$$

Here $\hat{x}$ and $\hat{p}$ are the coordinate and momentum operators of the particle and $\left\{\hat{q}_{j}, \hat{p}_{j}\right\}$ are the set of coordinate and momentum operators for the reservoir oscillators coupled linearly through the coupling constants $\kappa_{j}(j=1,2, \ldots)$. The potential $V(\hat{x}, t)$ is due to the external force field for the Brownian particle. The coordinate and momentum operators follow the usual commutation rules $\{\hat{x}, \hat{p}\}=i \hbar$ and $\left\{\hat{q}_{i}, \hat{p}_{j}\right\}=i \hbar \delta_{i j}$. Eliminating the bath degrees of freedom in the usual way we obtain the operator Langevin equation for the particle

$$
m \ddot{\hat{x}}+\int_{0}^{t} d t^{\prime} \gamma\left(t-t^{\prime}\right) \dot{\hat{x}}\left(t^{\prime}\right)+V^{\prime}(\hat{x}, t)=\hat{F}(t)
$$

where the noise operator $\hat{F}(t)$ and the memory kernel $\gamma(t)$ are given by

$$
\hat{F}(t)=\sum_{j}\left[\left\{\hat{q}_{j}(0)-\hat{x}(0)\right\} \kappa_{j} \cos \omega_{j} t+\kappa_{j}^{1 / 2} \hat{p}_{j}(0) \sin \omega_{j} t\right]
$$

and

$$
\gamma(t)=\sum_{j} \kappa_{j} \cos \omega_{j} t
$$

respectively with $\kappa_{j}=\omega_{j}^{2}$. On the basis of quantum mechanical average $\langle\ldots\rangle$ over the bath modes with coherent states and over the system mode with an arbitrary 
state Eq.(2.2) can be cast into the form of the generalized quantum Langevin equation [27, 28, 29, 30, 31, 32, 33, 34].

$m \ddot{x}+\int_{0}^{t} d t^{\prime} \gamma\left(t-t^{\prime}\right) \dot{x}\left(t^{\prime}\right)+V^{\prime}(x, t)=f(t)+Q\left(x,\left\langle\delta \hat{x}^{n}\right\rangle\right)$

where the quantum mechanical mean value of the position operator $\langle\hat{x}\rangle=x$. Here the quantum dispersion term $Q$ to the potential, is given by

$$
Q\left(x,\left\langle\delta \hat{x}^{n}\right\rangle\right)=V^{\prime}(x, t)-\left\langle V^{\prime}(\hat{x}, t)\right\rangle
$$

which by expressing $\hat{x}(t)=x(t)+\delta \hat{x}(t)$ in $V(\hat{x}, t)$ and using a Taylor series expansion around $x$ may be rewritten as

$$
Q\left(x,\left\langle\delta \hat{x}^{n}\right\rangle\right)=-\sum_{n \geq 2} \frac{1}{n !} V^{(n+1)}(x, t)\left\langle\delta \hat{x}^{n}\right\rangle
$$

Here $V^{(n+1)}$ the $(n+1)$ th derivative with respect to $x$. The calculation of $Q$ rests on the quantum correction terms $\left\langle\delta \hat{x}^{n}\right\rangle$ which can be calculated order by order by solving a set of quantum correction equations(as discussed in the later part of this section). Furthermore the quantum mechanical mean Langevin force is given by

$f(t)=\sum_{j}\left[\left\langle\hat{q}_{j}(0)\right\rangle-\langle\hat{x}(0)\rangle \kappa_{j} \cos \omega_{j} t+\kappa_{j}^{1 / 2} \hat{p}_{j}(0) \sin \omega_{j} t\right]$

which must satisfy noise characteristics of the bath at equilibrium ,

$$
\begin{aligned}
\langle f(t)\rangle_{S} & =0 \\
\left\langle f(t) f\left(t^{\prime}\right)\right\rangle_{S} & =\frac{1}{2} \sum_{j} \kappa_{j} \hbar \omega_{j}\left(\operatorname{coth} \frac{\hbar \omega_{j}}{2 k T}\right) \cos \omega_{j}\left(t-t^{\prime}\right)
\end{aligned}
$$

Eq.(2.10) expresses the quantum fluctuationdissipation relation. The above conditions Eq.(2.9)Eq.(2.10) can be fulfilled provided the initial shifted co-ordinates $\left\{\left\langle\hat{q}_{j}(0)\right\rangle-\langle\hat{x}(0)\rangle\right\}$ and momenta $\left\langle\hat{p}_{j}(0)\right\rangle$ of the bath oscillators are distributed according to the canonical thermal Wigner distribution [37, 38] of the form

$$
\begin{aligned}
& P_{j}\left(\left[\left\langle\hat{q}_{j}(0)\right\rangle-\langle\hat{x}(0)\rangle\right],\left\langle\hat{p}_{j}(0)\right\rangle\right) \\
= & N \exp \left\{-\frac{\frac{1}{2}\left\langle\hat{p}_{j}(0)\right\rangle^{2}+\frac{1}{2} \kappa_{j}\left[\left\langle\hat{q}_{j}(0)\right\rangle-\langle\hat{x}(0)\rangle\right]^{2}}{\hbar \omega_{j}\left[\bar{n}\left(\omega_{j}\right)+\frac{1}{2}\right]}\right\}
\end{aligned}
$$

so that the statistical averages $\langle\ldots\rangle_{s}$ over the quantum mechanical mean value $O_{j}$ of the bath variables are defined as

$$
\left\langle O_{j}\right\rangle_{s}=\int O_{j} P_{j} d\left\langle\hat{p}_{j}(0)\right\rangle d\left\{\left\langle\hat{q}_{j}(0)\right\rangle-\langle\hat{x}(0)\rangle\right\}
$$

Here $\bar{n}(\omega)$ is given by Bose-Einstein distributions $\left(e^{\frac{\hbar \omega}{k T}}-1\right)^{-1} . P_{j}$ is the exact solution of Wigner equation for harmonic oscillator [37, 38] and forms the basis for description of the quantum noise characteristics of the bath kept in thermal equilibrium at temperature $T . N$ is the normalization constant. In the continuum limit the fluctuation-dissipation relation (2.10) can be written as

$$
\begin{aligned}
& \left\langle f(t) f\left(t^{\prime}\right)\right\rangle_{s} \\
= & \frac{1}{2} \int_{0}^{\infty} d \omega \kappa(\omega) \rho(\omega) \hbar \omega \operatorname{coth}\left(\frac{\hbar \omega}{2 k T}\right) \cos \omega\left(t-t^{\prime}\right)
\end{aligned}
$$

where we have introduced the density of the modes $\rho(\omega)$. Since we are interested in the Markovian limit in the present context, we assume $\kappa(\omega) \rho(\omega)=\frac{2}{\pi} \gamma$, and Eq.(2.13) then yields

$$
\left\langle f(t) f\left(t^{\prime}\right)\right\rangle_{s}=2 D_{q} \delta\left(t-t^{\prime}\right)
$$

with

$$
D_{q}=\frac{1}{2} \gamma \hbar \omega_{0} \operatorname{coth} \frac{\hbar \omega_{0}}{2 k T}
$$

(The passage from Eq.(2.13) to Eq.(2.14) is given in the appendix A)

$\omega_{0}$ refers to the static frequency limit. Furthermore from Eq. (2.4) in the continuum limit we have

$$
\gamma\left(t-t^{\prime}\right)=\gamma \delta\left(t-t^{\prime}\right)
$$

$\gamma$ is the dissipation constant in the Markovian limit. In this limit Eq.(2.5) therefore reduces to

$$
m \ddot{x}+\gamma \dot{x}+V^{\prime}(x, t)=f(t)+Q\left(x,\left\langle\delta \hat{x}^{n}\right\rangle\right)
$$

In order to consider the activated processes in a bistable potential with a fluctuating barrier height we consider the potential of the form

$$
V(x, t)=U(x)+g(x) \xi(t)
$$

where $U(x)$ is a bistable potential $\left(-\frac{a}{2} x^{2}+\frac{b}{4} x^{4}, a, b\right.$ being constants ) with a barrier at the metastable point $x=0$ and two stable points at $x= \pm\left(\frac{a}{b}\right)^{\frac{1}{2}}$. The fluctuations in 
the potential are driven by an Ornstein-Uhlenbeck noise process

$$
\dot{\xi}(t)=-\frac{\xi(t)}{\tau}+\frac{\sqrt{2 \sigma^{2}}}{\tau} \eta(t)
$$

where $\eta(t)$ is zero mean $\delta$-correlated Gaussian noise. The stochastic process $\xi(t)$, can be characterized by the following set of equations. The probability function $\bar{\rho}(\xi)$, the variance $\sigma^{2}$ and the correlation function of the noise are given

$$
\bar{\rho}(\xi)=\sqrt{2 \pi \sigma^{2}} \exp \left(-\frac{\xi^{2}}{2 \sigma^{2}}\right)
$$

where

$$
\sigma^{2}=\int_{-\infty}^{+\infty} \xi^{2} \bar{\rho}(\xi) d \xi
$$

and

$$
\langle\xi(t) \xi(0)\rangle=\sigma^{2} \exp \left(-\frac{|t|}{\tau}\right)
$$

respectively. The barrier can also be subjected to dichotomic fluctuation 36$], \xi(t)=\{-\alpha, \beta\}$ that flips between two values with flipping rate $\mu_{\alpha}$ and $\mu_{\beta}$ respectively. This process can also be taylored as zero mean valued, exponentially correlated and with a Gaussian distribution.

\section{B. Overdamped limit}

To proceed further we now confine ourselves to overdamped condition so that the quantum Langevin equation in one variable takes the following form:

$$
\gamma \dot{x}=a x-b x^{3}+g^{\prime}(x) \xi(t)+f(t)+Q\left(x,\left\langle\delta \hat{x}^{n}\right\rangle\right)
$$

The above classical-like stochastic differential equation contains the essential quantum features, through the terms $f(t)$ which represent the quantum noise of the heat bath and the another term $Q\left(x,\left\langle\delta \hat{x}^{n}\right\rangle\right)$ which essentially arises due to nonlinear part of the system potential. The nonlinearity and the quantum effects are entangled in the latter quantity modifying the classical part of the potential. Thus the classical potential force $-V^{\prime}(x, t)$ is modified by the quantum dispersion. In absence of quantum dispersion term $Q\left(x,\left\langle\delta \hat{x}^{n}\right\rangle\right)$ and with $D_{q} \rightarrow \gamma k T$ as one approaches the classical limit $\left(k T \gg \hbar \omega_{0}\right)$, the quantum Langevin equation reduces to classical one. A probabilistic description of the system Eq. (2.23) is provided by the time-dependent probability distribution function $\bar{P}(x, \xi, t)$. For a finite correlation time of the noise driving the barrier to fast fluctuations it is possible 21] to go over to an approximate (exact in the limit $\tau \rightarrow 0$ ) description which allows one to transform externally driven Langevin equation Eq.(2.23) into an autonomous Langevin equation for the position variable $x$. The corresponding equation for probability density function $P(x, t)$ is given by

$$
\begin{aligned}
\frac{\partial P(x, t)}{\partial t} & =-\frac{\partial}{\partial x}[\kappa(x, \tau) f(x)] P(x, t) \\
& +\sigma^{2} \frac{\partial}{\partial x}\left[\kappa(x, \tau) g^{\prime}(x) \frac{\partial}{\partial x} \kappa(x, \tau) g^{\prime}(x)\right] P(x, t) \\
& +D_{q} \frac{\partial}{\partial x}\left[\kappa(x, \tau) \frac{\partial}{\partial x} \kappa(x, \tau)\right] P(x, t)
\end{aligned}
$$

where $\gamma$ is assumed to unity, $\kappa(x, \tau)=$ $\left[1-\tau g^{\prime}(x)\left(\frac{f(x)}{g^{\prime}(x)}\right)^{\prime}\right]^{-1}$ and $f(x)=-U^{\prime}(x)+$ $Q\left(x,\left\langle\delta \hat{x}^{n}\right\rangle\right)$. The quantum nature of the above Fokker-Planck equation governing evolution of $P(x, t)$ is manifested through the quantum correction to the potential term and the quantum diffusion coefficient characterizing the thermal bath.

The quantity of special interest here is the mean thermally activated escape time of the particle from the well whose barrier is subjected to fluctuation. The particle is governed by the stochastic dynamics Eq.(2.24). It is important to emphasize that the typical mean escape time should be much larger than the time scale of the deterministic quantum dynamics $\dot{x}=-U^{\prime}(x)+Q\left(x,\left\langle\delta \hat{x}^{n}\right\rangle\right)$ for nonzero fluctuation of the potential well, and it is further required that the strengths of thermal noise and barrier fluctuation must be small in comparison with the barrier height. The solution of Eq.(2.24) in the stationary state reads as

$$
\begin{gathered}
P_{s}(x, \tau)=\frac{\left[1-\tau g^{\prime}(x)\left(\frac{f(x)}{g^{\prime}(x)}\right)^{\prime}\right]}{D_{q}^{\frac{1}{2}}\left(1+\frac{\sigma^{2}}{D_{q}} g^{\prime}(x)^{2}\right)^{\frac{1}{2}}} \\
\times \exp \left(\int_{0}^{x} f(y) \frac{\left[1-\tau g^{\prime}(y)\left(\frac{f(y)}{g^{\prime}(y)}\right)^{\prime}\right]}{D_{q}\left(1+\frac{\sigma^{2}}{D_{q}} g^{\prime}(y)^{2}\right)} d y\right)
\end{gathered}
$$

The stationary probability distribution function $P_{s}(x, \tau)$ of the stochastic process $x(t)$, which is the quantum mechanical mean position, is bimodal in nature with nonzero probability current at the barrier top even at zero temperature characterizing the zero point contribution of the thermal bath (as $\left.T \rightarrow 0, D_{q} \rightarrow \hbar \omega_{0}\right)$. $P(x, \tau)$ is additionally modified over its classical nature by a quantum contribution characterizing a correction due to anharmonic part of the system potential.

To proceed further it is necessary to find out the quantum correction term $Q\left(x,\left\langle\delta \hat{x}^{n}\right\rangle\right)$ more explicitly. To this end we return to the overdamped operator equation (2.2) and use $\hat{x}(t)=x(t)+\delta \hat{x}(t)$, where $x(t)(=\langle\hat{x}(t)\rangle)$ is the quantum mechanical mean value of the operator $\hat{x}$. By 
construction $[\delta \hat{x}, \delta \hat{p}]=i \hbar$ and $\langle\delta \hat{x}\rangle=0$. We then obtain the quantum correction equation in the overdamped limit after quantum mechanical averaging with the coherent states over the bath operators as

$$
\gamma \delta \dot{\hat{x}}+V^{\prime \prime}(x, t) \delta \hat{x}+\sum_{n \geq 2} \frac{1}{n !} V^{n+1}(x, t)\left(\delta \hat{x}^{n}-\left\langle\delta \hat{x}^{n}\right\rangle\right)=0
$$

With the help of operator equation (2.26) we obtain the equations for $\left\langle\delta \hat{x}^{n}\right\rangle$

$$
\begin{aligned}
\frac{d}{d t}\left\langle\delta \hat{x}^{2}\right\rangle=- & \frac{1}{\gamma}\left[2 V^{\prime \prime}(x, t)\left\langle\delta \hat{x}^{2}\right\rangle+V^{\prime \prime \prime}(x, t)\left\langle\delta \hat{x}^{3}\right\rangle\right] \\
\frac{d}{d t}\left\langle\delta \hat{x}^{3}\right\rangle= & -\frac{1}{\gamma}\left[3 V^{\prime \prime}(x, t)\left\langle\delta \hat{x}^{3}\right\rangle+\frac{3}{2} V^{\prime \prime \prime}(x, t)\left\langle\delta \hat{x}^{4}\right\rangle\right] \\
+ & {\left[\frac{3}{2 \gamma} V^{\prime \prime \prime}(x, t)\left\langle\delta \hat{x}^{2}\right\rangle^{2}\right] }
\end{aligned}
$$

and so on. To take into account of the leading order contribution $\left\langle\delta \hat{x}^{2}\right\rangle$ explicitly we may write

$$
d\left\langle\delta \hat{x}^{2}\right\rangle=-\frac{2}{\gamma} V^{\prime \prime}(x, t)\left\langle\delta \hat{x}^{2}\right\rangle d t
$$

The overdamped deterministic motion on the other hand gives $\gamma d x=-V^{\prime}(x, t) d t$ which when used in Eq.(2.29) yields after integration

$$
\left\langle\delta \hat{x}^{2}\right\rangle=\Delta_{q}\left[V^{\prime}(x, t)\right]^{2}
$$

$\Delta_{q}$ is the quantum correction parameter, as given by $\Delta_{q}=\frac{\left\langle\delta \hat{x}^{2}\right\rangle_{x_{c}}}{\left[V^{\prime}\left(x_{c}, t\right)\right]^{2}}, x_{c}$ being a given quantum mechanical mean position.

\section{RESONANT ACTIVATION IN THE QUANTUM SYSTEM}

We are now in a position to analyze the quantum resonant activation. In our present problem the fluctuating part of the potential associated with harmonic term is assumed to be of the form $g(x)=\frac{x^{2}}{2}$ and $x= \pm \sqrt{\frac{a}{b}}$ and $x=0$ are the absolute minima and maximum of the potential $U(x)$ respectively. Having known the stationary distribution Eq. 2.25) along with the quantum correction in $f(x)$ the calculation of stochastic dynamics is straightforward. This can be obtained using the standard result on mean first passage time [26] as

$$
\langle T\rangle=\int_{-\sqrt{\frac{a}{b}}}^{0} \frac{d x}{D(x, \tau) P_{s}(x)} \int_{-\infty}^{x} P_{s}(x) d y
$$

where $D_{\text {eff }}(x, \tau)=D_{q}\left(1+R g^{\prime}(x)^{2}\right)\left[1-\tau g^{\prime}(x)\left(\frac{f(x)}{g^{\prime}(x)}\right)^{\prime}\right]$ to give

$$
\langle T\rangle=\frac{2 \pi \sqrt{1+2 \tau\left(a+\Delta_{1}\right)}}{a \sqrt{2\left(1-\Delta_{2}\right)}} \exp \left[\frac{\Delta V_{e f f}(R, \tau)}{D_{q}}\right]
$$

Here $\Delta_{1}$ and $\Delta_{2}$ both are the leading order quantum correction terms as given by

$$
\begin{gathered}
\Delta_{1}=\left[-g^{\prime}(x)\left(\frac{Q\left(x,\left\langle\delta \hat{x}^{n}\right\rangle\right)}{g^{\prime}(x)}\right)\right]_{x=-\sqrt{\frac{a}{b}}} \\
\Delta_{2}=\left[Q^{\prime}\left(x,\left\langle\delta \hat{x}^{n}\right\rangle\right)\right]_{x=-\sqrt{\frac{a}{b}}}
\end{gathered}
$$

and $R=\frac{\sigma^{2}}{D_{q}} ; \Delta V_{e f f}(R, \tau)$ is the effective barrier height of the following form

$$
\begin{aligned}
& \Delta V_{\text {eff }}(R, \tau) \\
= & -\int_{-\sqrt{\frac{a}{b}}}^{0} \frac{\left(-U^{\prime}(x)+Q\right)\left[1+\tau g^{\prime}(x)\left(\frac{-U^{\prime}(x)+Q}{g^{\prime}(x)}\right)\right] d x}{\left(1+R g^{\prime}(x)^{2}\right)}
\end{aligned}
$$

Eq. 3.2 is the central analytical result of this paper. To analyse the theoretical results on the essential features of the activated escape following quantum stochastic dynamics over a fluctuating potential well we now resort to numerical simulation of the Eqs.(2.23),(2.19) and the quantum correction equations (2.27) and (2.28) simultaneously using standard Heun's algorithom. A very small time step $(\triangle t) 0.001$ for numerical integration has been used. In our simulation we follow the dynamics of each particle starting in the left well at $x=-\sqrt{\frac{a}{b}}$ till it arrives at the barrier top at $x=0$. The first passage time being a statistical quantity due to the random force we calculate the statistical average of the first passage time over 1,000 trajectories. We present the numerical results in Fig.1 to Fig.4 for different parameters such as temperatures, barrier height of the potential and noise strength of the Ornstein-Unhenbeck noise process. All the curves exhibit minimum at optimal $\tau$ values. Increasing strength of thermal or non-thermal noise results in enhancement of escape rate and the minima are shifted towards the origin. Physically this implies that with increase in $D_{q}$ or $\sigma^{2}$, the escape time over the barrier changes as a result of which the switching time of the barrier fluctuations matches the order of escape time at lower $\tau$ values. In order to examine the influence of the barrier height on resonant activation we plot in Fig.3 the variation of mean escape time as function of $\tau$ for several values of barrier heights. Increase in the barrier height results in enhancement of escape time and the minima are shifted to higher $\tau$ values.

We find that the barrier height Eq. 3.5 monotonically decreases to a limiting value with increasing $\tau$. Due to ${ }^{-2}$ the presence of system nonlinearity the barrier height is slightly modified by an added slope. It is thus expected that during the temporary stay of the particle close to a minimum it makes unsuccessful attempts to escape and once the escape takes place it should occur at a slower rate in quantum case due to added slope to the effective 
potential than the corresponding classical case. However, our theoretical and numerical calculations reveal that the mean escape time is lower in magnitude for the quantum particle at low temperature and the difference becomes insignificant at higher temperature. This is shown in the Fig.4. This behaviour can be interpreted in terms of an interplay between the quantum diffusion coefficient $D_{q}$ and the quantum correction due to system nonlinearity appearing in $V_{e f f}(R, \tau)$. When the temperature of the system is very low, i. e., in the vacuum limit or in the deep tunneling region the anharmonic terms in the potential do not contribute significantly. On the other hand as temperature of the system increases significantly, $D_{q}$ increases resulting in decrease of the effective potential and hence $D_{q}$ and $Q$ compete to cancel the effect of each other at higher temperature. Finally in Fig.5 we make a comparison between the numerical simulation and the corresponding theoretical result. The theoretical result agrees fairly well with our numerical result.

In addition to the barrier height the prefactor is also affected by the quantum correction term. The approximate theoretical mean escape time Eq. (3.2) implies that both the barrier height and the frequency factor have an important role to play with the activated escape process. We are now in a position to point out that the thermally activated resonance phenomenon is controlled by three different time scales as emphasized earlier [19]. The three relavent time scales are the corresponding time of barrier fluctuation $(0 \ll \tau \ll \infty)$, typical escape time $\hat{T}$, which is much larger than the time scale describing deterministic motion $\dot{x}=-U^{\prime}(x)+Q^{\prime}\left(x,\left\langle\delta \hat{x}^{n}\right\rangle\right)$, the third time scale being $T_{a}$ of the escape attempt $\left(T_{a} \ll \hat{T}\right)$.

\section{CONCLUSION}

Based on the theoretical study and numerical simulation of quantum stochastic dynamics in a double-well system with a fluctuating barrier under influence of a Gaussian color noise, we have examined the phenomenon of resonant activation. The governing equations are classical looking in form but quantum mechanical in their content. A key point of the present analysis is to describe the thermal bath in terms of a canonical thermal Wigner distribution of harmonic oscillators. This distribution remain positive definite even at absolute zero signifying a pure state and allows us to look for the external noiseinduced resonance when the generic quantum effects in the system make their presence felt. We have shown that quantization significantly enhances resonant activation at low temperature due to tunneling. For higher temperature as well as for stronger noise strength the resonance effects get more pronounced. Since tunneling accompanies activation, it is expected that the resonant activation can be observed even at absolute zero, where the resonance effect essentially is due to the vacuum field.

\section{APPENDIX A: (THE PASSAGE FROM EQ.(2.13)} TO EQ. (2.14)

We start from basic definition(Louisell p-426) [39]

$$
2 D_{q}=\frac{1}{2 \Delta t} \int_{t}^{t+\Delta t} d t \int_{t}^{t+\Delta t} d t^{\prime}\left\langle f(t) f\left(t^{\prime}\right)\right\rangle_{s}
$$

Using Eq. 2.13) in Eq. A1 yields

$$
\begin{aligned}
2 D_{q}= & \frac{1}{2 \Delta t} \int_{0}^{\infty} d \omega \kappa(\omega) \rho(\omega) \hbar \omega \operatorname{coth}\left(\frac{\hbar \omega}{2 k T}\right) \\
& \times \int_{t}^{t+\Delta t} d t \int_{t}^{t+\Delta t} d t^{\prime} \cos \omega\left(t-t^{\prime}\right)
\end{aligned}
$$

Explicit integration over time gives

$$
2 D_{q}=\frac{1}{2 \Delta t} \int_{0}^{\infty} d \omega \kappa(\omega) \rho(\omega) \hbar \omega \operatorname{coth}\left(\frac{\hbar \omega}{2 k T}\right) \quad I(\omega, \Delta t)
$$

where

$$
I(\omega, \Delta t)=\frac{4}{w^{2}} \quad \sin ^{2} \frac{\omega \Delta t}{2}
$$

Putting $\kappa(\omega) \rho(\omega)=\frac{2}{\pi} \gamma$, we obtain

$$
2 D_{q}=\frac{\gamma}{\Delta t \pi} \int_{0}^{\infty} d \omega \hbar \omega \operatorname{coth}\left(\frac{\hbar \omega}{2 k T}\right) \frac{\sin ^{2} \frac{\omega \Delta t}{2}}{\left(\frac{\omega}{2}\right)^{2}}
$$

Following Louisell(p -426) 39] we have under Markovian condition, the correlation time $\tau_{c} \ll \Delta t$, the coarse-grain time(over which the probability distribution function evolves)

Thus as $\Delta t \rightarrow \infty$ (in scale of $\tau_{c}$ which goes to zero) the function $\frac{\sin ^{2} \frac{\omega \Delta t}{2}}{\left(\frac{\omega}{2}\right)^{2}}$ oscillates violently so that one takes the slowly varying quantity $\left[\hbar \omega \operatorname{coth} \frac{\hbar \omega}{2 k T}\right]$ out of the integration over frequency with an average value $\hbar \omega_{0} \operatorname{coth} \frac{\hbar \omega_{0}}{2 k T}$, $\omega_{0}$ be an average static frequency. Since the integral $\int_{\infty}^{\infty} \frac{\sin ^{2} x \Delta t}{x^{2}} d x=\pi \Delta t$ it follows immediately from Eq. A5

$$
2 D_{q}=\gamma \hbar \omega_{0} \operatorname{coth}\left(\frac{\hbar \omega_{0}}{2 k T}\right)
$$

as given in Eq. (2.15)

Again starting from Eq.(2.13), we use the same argument as before to have

$$
\begin{aligned}
& \left\langle f(t) f\left(t^{\prime}\right)\right\rangle_{s} \\
& =\frac{1}{2} \int_{0}^{\infty} d \omega \kappa(\omega) \rho(\omega) \hbar \omega \operatorname{coth}\left(\frac{\hbar \omega}{2 k T}\right) \cos \omega\left(t-t^{\prime}\right)
\end{aligned}
$$

and we use 


$$
\int_{0}^{\infty} d \omega \cos \omega \tau=\pi \delta(\tau)
$$

to obtain

$$
\begin{aligned}
& \left\langle f(t) f\left(t^{\prime}\right)\right\rangle_{s} \\
& =\frac{1}{2} \int_{0}^{\infty} d \omega\left[\frac{2}{\pi} \gamma\right] \hbar \omega \operatorname{coth}\left(\frac{\hbar \omega}{2 k T}\right) \cos \omega\left(t-t^{\prime}\right) \\
& =\frac{\gamma \hbar \omega_{0}}{\pi} \operatorname{coth}\left(\frac{\hbar \omega_{0}}{2 k T}\right) \pi \delta\left(t-t^{\prime}\right) \\
& =\gamma \hbar \omega_{0} \operatorname{coth}\left(\frac{\hbar \omega_{0}}{2 k T}\right) \delta\left(t-t^{\prime}\right)
\end{aligned}
$$

Therefore from Eq. A66) and Eq. (A7) we have

$$
\left\langle f(t) f\left(t^{\prime}\right)\right\rangle_{s}=2 D_{q} \delta\left(t-t^{\prime}\right)
$$

which is Eq. (2.14).

Thus the derivation within Markovian approximation clearly depends on the time scale separation. The results are valid even at absolute zero as emphasized by Louisell.

\section{Acknowledgments}

Thanks are due to the Council of Scientific and industrial research, Govt. of India, for partial financial support.
[1] R. Benzi, G. Parisi, A. Sutera, and A. Vulpiani, Tellus 34, 10 (1982); R. Benzi, A. Sutera, and A. Vulpiani, J. Phys. A 14 L 453 (1981)

[2] B. McNamara and K. Wiesenfeld, Phys. Rev. A, 39, 4854 (1988); L. Gammaitoni, F. Marchesoni, E. MenichellaSaetta and S. Santucci, Phys. Rev. Lett. 62, 349 (1989).

[3] B. McNamara, K. Wiesenfeld and R. Roy, Phys. Rev. Lett. 60, 2626 (1988)

[4] L. Gammaitoni, P. Hänggi, P. Jung and F.Marchesoni, Rev. Mod. Phys., 70, 223(1998)

[5] F. Julicher, A. Ajdari and J. Prost, Rev. Mod. Phys. 69, 1269 (1997).

[6] M. O. Magnasco, Phys. Rev. Lett. 71, 1477 (1993), C. R. Doering, W. Horsthemke and J. Riordan, Phys. Rev. Lett. 72, 2984 (1994).

[7] H. Linke, T. E. Humphrey, A. Lfgren, A. O. Sushkov, R. Newbury, R. P. Taylor, and P. Omling, Science 286, 2314 (1999); L. Q. Zhou, X. Jia and Q. Ouyang, Phys. Rev. Lett. 88, 138301(2002)

[8] J. Garcia-Ojalvo, A. Hernandez-Machado and J. M. Sancho, Phys. Rev. Lett. 71, 1542 (1993)

[9] A. Becker and L. Kramer, Phys. Rev. Lett. 73, 955 (1994), A. Becker and L. Kramer, Physica D 90, 408(1995)

[10] J. M. R. Parrondo, C. Van den Broeck, J. Buceta and F. J. de la Rubia, Physica A 224, 153(1996)

[11] A. A. Zaikin and L. Schimansky-Geier, Phys. Rev. E. 58, 4355(1998)

[12] J. Buceta, M. Ibanes, J. M. Sancho and K. Lindenberg, Phys. Rev. E 67, 021113(2003)

[13] S. Dutta, S. S. Riaz and D. S. Ray, Phys. Rev. E 71, 036216 (2005)

[14] C. R. Doering and J. C. Gadoua, Phys. Rev. Lett. 69, 2318 (1992)

[15] M. Bier and R. D. Astumian, Phys. Rev. Lett. 71, 1649 (1993); U. Zürcher and C. R. Doering, Phys. Rev. E 47, 3862 (1993)

[16] C. Van den Broeck, Phys. Rev. E 47, 4579 (1994)

[17] J. J. Brey and J. Casado-Pascual, Phys. Rev. E. 50, 116 (1994); O. Flomenbom and J. Klafter, Phys. Rev. E. 69,
051109 (2004)

[18] T. D. Shepherd and R. Hernandez J. Chem. Phys. 115, 2430 (2001); A. M. Berezhkovskii, A. Szabo, G. H. Weiss, and H. Zhou J. Chem. Phys. 111, 9952 (1999)

[19] M. Boqun̈á, J. M. Porrá, J. Masoliver and K. Lindenberg, Phys. Rev. E 57, 003990 (1998)

[20] M. Marchi, F. Marchesoni, L. Gammaitoni, E. Menichella -Saetta, and S. Santucci Phys. Rev. E 54, 3479 (1995)

[21] P. Hänggi, Chem. Phys., 180157 (1994); R. F. Fox, Phys. Rev. A. 34, R4525 (1986); Y. Jia and J. R. Li Phys. Rev. E. 53, 5786 (1996); X. Luo and S. Zhu, Phys. Rev. E 67, 021104 (2003)

[22] R. N. Mantegna and B. Spagnolo, Phys. Rev. Lett. 84, 3025 (2000)

[23] J. Maddox , Nature(London) 359, 771 (1992).

[24] J. Wang and P. Wolynes, Comput. Phys. 180141 (1994)

[25] J. M. Martinis, M. H. Devoret and J. Clarke, Phys. Rev. Lett. 55, 1543 (1985)

[26] R. L. Straonovich, Topics in the Theory of Random Noise, Vol. 1 (Gordon and Breach, New York, 1963); K. Lindenberg and B.J. West, J. Stat. Phys. 42, 201 (1986); J. Masoliver, B.J. West, and K. Lindenberg, Phys. Rev. A 35, 3086 (1987); D. Mei, G. Xie, Li Cao, and D. Wu, Phys. Rev. E 59, 3880 (1999)

[27] D. Banerjee, B. C. Bag, S. K. Banik and D. S. Ray, Phys. Rev. E 65, 021109 (2002).

[28] D. Barik and D. S. Ray, J. Chem. Phys. 119, 12973 (2003).

[29] D. Barik, S. K. Banik and D. S. Ray, J. Chem. Phys. 119, 680 (2003).

[30] D. Banerjee, B. C. Bag, S. K. Banik and D. S. Ray, J. Chem. Phys. 120, 8960 (2004).

[31] D. Barik and D. S. Ray, J. Chem. Phys. 121, 1681 (2004).

[32] P. K. Ghosh, D. Barik and D. S. Ray, Phys. Rev. E 71, 041107 (2005)

[33] P. K. Ghosh, D. Barik and D. S. Ray, Physics Letters A 34212 (2005).

[34] D. Barik and D. S. Ray, J. Stat. Phys. (2005) To be published.

[35] R. Zwanzig, J. Stat. Phys.9, 215 (1973). 
[36] A. Mielke, Ann. Phys. 4, 476 (1995); J. M. Sancho, Phys. Rev. A 31, R3523 (1985); J. M. Gutirrez, A. Iglesias, and M. A. Rodrguez, Phys. Rev. E. 48, 2507 (1993)

[37] E. P. Wigner, Phys. Rev. 40, 749 (1932).

[38] M. Hillery, R. F. O'Connell, M. O. Scully and E. P. Wigner, Phys. Reps. 106, 121 (1984).

[39] W. H. Louisell, Quantum Statistical Properties of Radiation, (John Wiley and Sons, New York, 1990);

\section{Figure Captions}

Fig.1. A plot of mean escape time vs $\tau$ using Gaussian colour noise for different temperatures [(i) $T=0.1$ (dotted line), (ii) $T=1.0$ (dashed line), (iii) $T=2.0$ (solid line)] and the other parameter set $a=0.5, b=0.005$ and $\sigma^{2}=1.0$.

Fig.2. A plot of mean escape time vs $\tau$ using Gaussian colour noise for different $\sigma^{2}\left[(\mathrm{i}) \sigma^{2}=0.5\right.$ (dashed line), (ii) $\sigma^{2}=1.0$ (dotted line), (iii) $\sigma^{2}=1.5$ (solid line)] and the other parameter set $a=0.5, b=0.005$ and $T=1.0$.
Fig.3. A plot of mean escape time vs $\tau$ using Gaussian colour noise for different barrier heights [(i) $a=0.3$ (dashed line), (ii) $a=0.5$ (dotted line), (iii) $a=0.7$ (solid line)] and the other parameter set $b=0.005, \sigma^{2}=1.0$ and $T=1.0$.

Fig.4. A comparison between classical(dotted line) and quantum (solid line) mean escape time describing resonant activation phenomenon using Gaussian colour noise for the parameter set $a=0.5, b=0.005$ and $\sigma^{2}=1.0$ at two different temperatures $T=1.5$ and $T=0.1$.

Fig.5. A comparison between numerical(dashed dot dot line and dashed line) and analytical (solid and dotted line) results using Gaussian colour noise for different $\sigma^{2}$ [(i) $\left.\sigma^{2}=0.5\right)$ (solid and dashed dot dot line), (ii) $\sigma^{2}=1.5$ (dotted and dashed line)] and the other parameter set $a=0.5, b=0.005$ and $T=1.5$. 


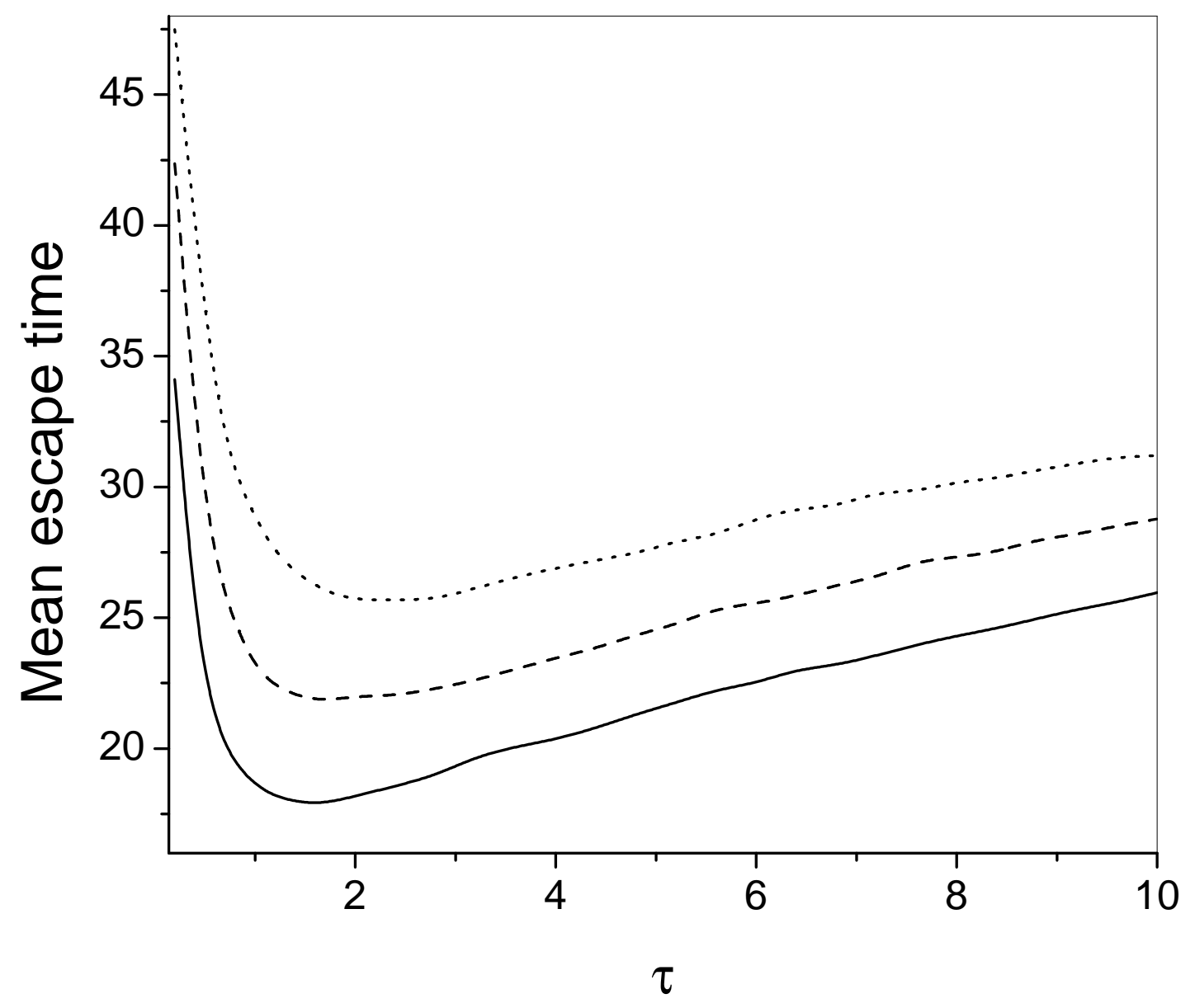

Fig.1 


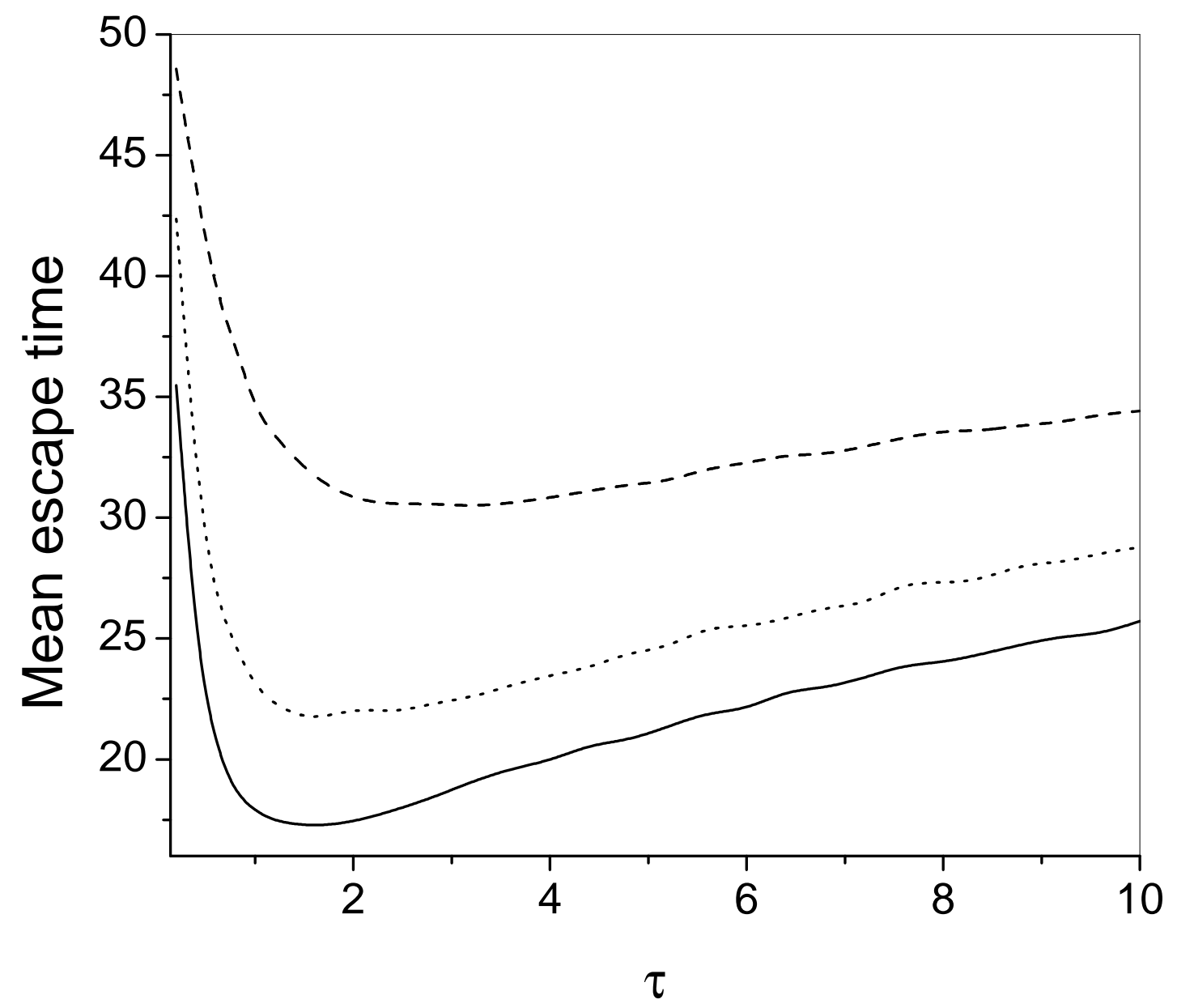

Fig.2 


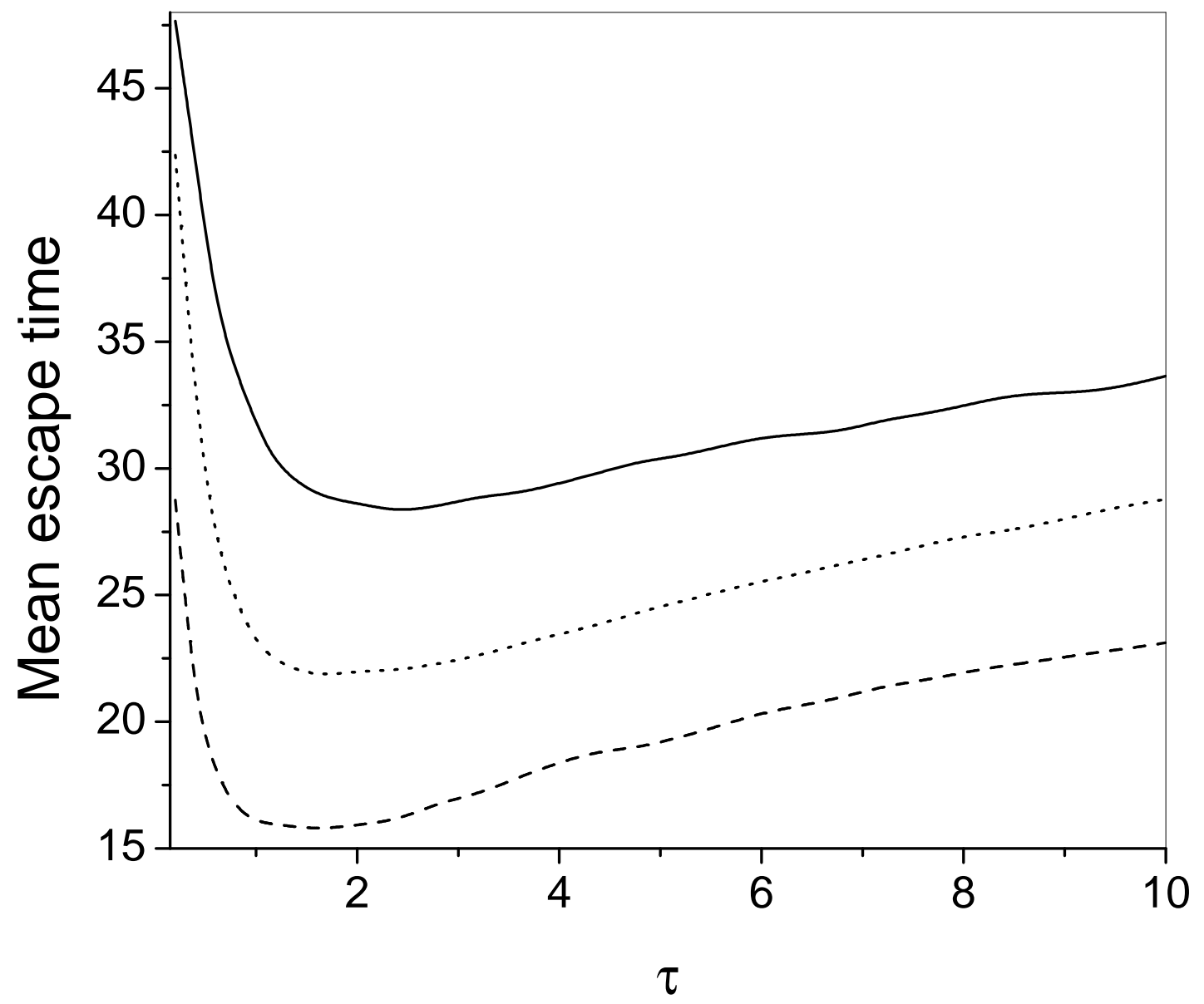

Fig.3 


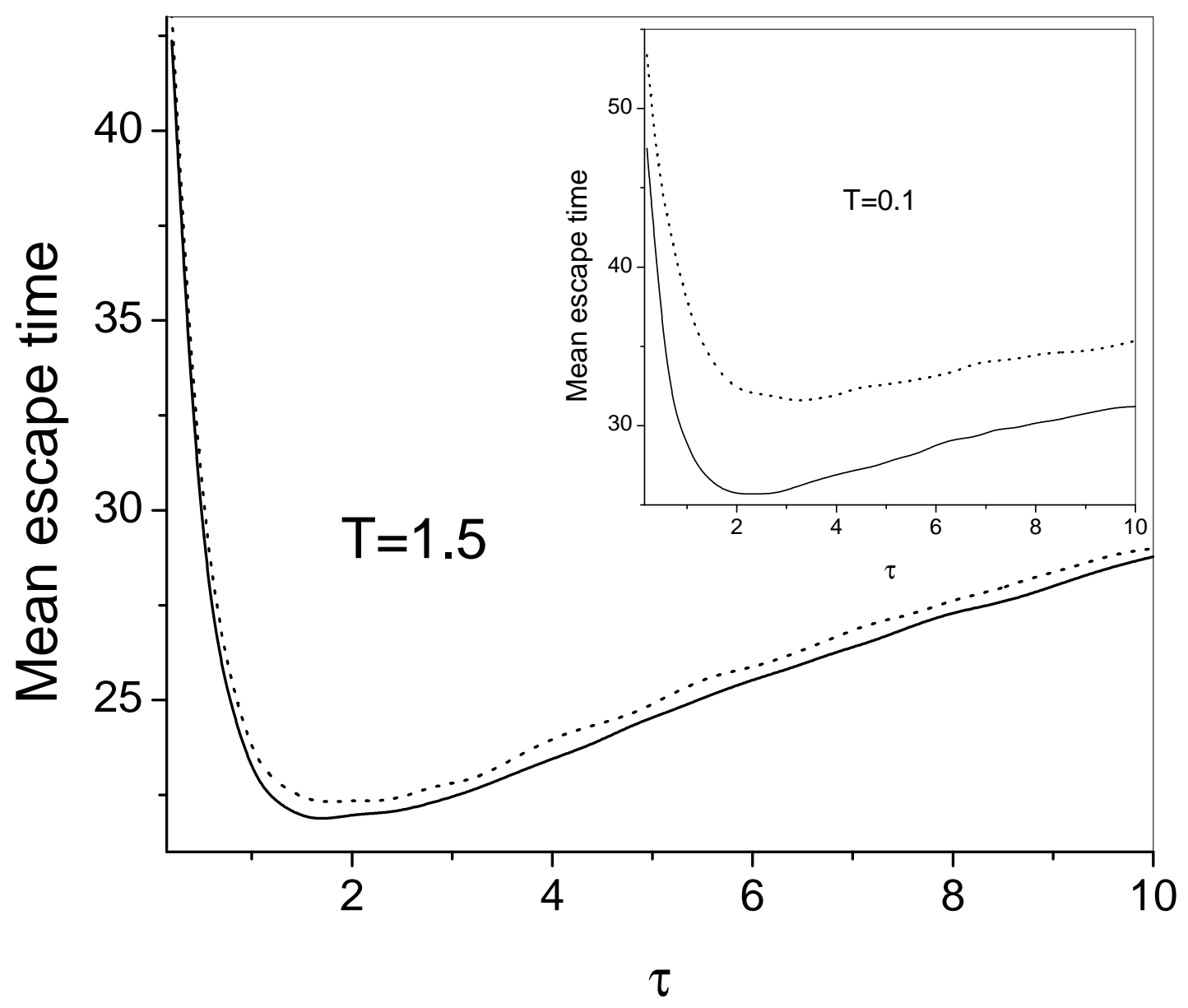

Fig.4 


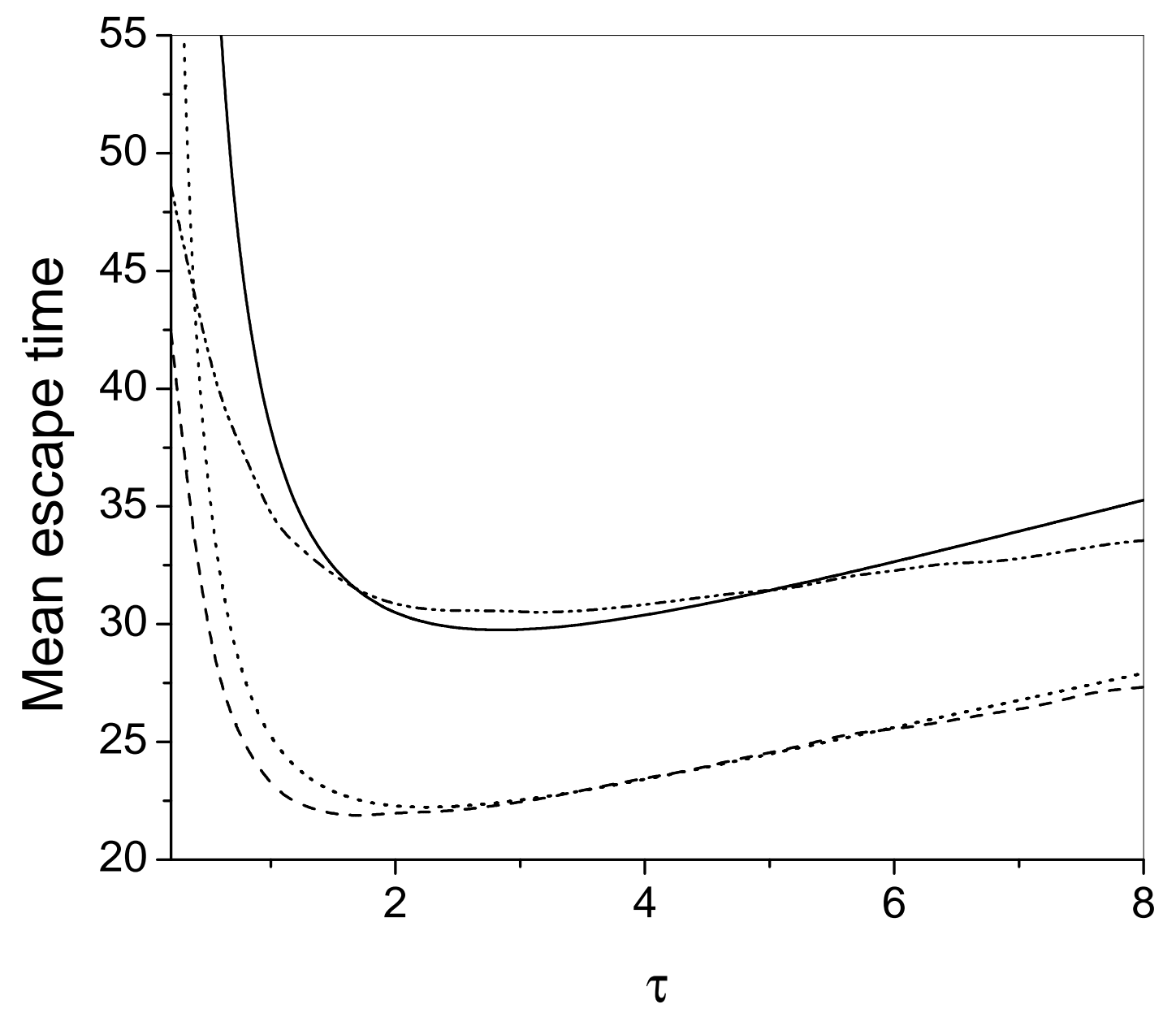

Fig.5 